Formation en anglais de spécialité dans les départements informatiques des IUT : choix des matériaux linguistiques et didactisation

\title{
Arlette Dechet
}

\section{OpenEdition}

\section{Journals}

Édition électronique

URL : http://journals.openedition.org/asp/4367

DOI : $10.4000 /$ asp. 4367

ISSN : 2108-6354

\section{Éditeur}

Groupe d'étude et de recherche en anglais de spécialité

Édition imprimée

Date de publication : 1 mars 1993

Pagination : 139-187

ISSN : 1246-8185

\section{Référence électronique}

Arlette Dechet, « Formation en anglais de spécialité dans les départements informatiques des IUT: choix des matériaux linguistiques et didactisation », ASp [En ligne], 1 | 1993, mis en ligne le 25 avril 2014, consulté le 30 avril 2019. URL : http://journals.openedition.org/asp/4367 ; DOI : 10.4000/ asp.4367

Ce document a été généré automatiquement le 30 avril 2019.

Tous droits réservés 


\title{
Formation en anglais de spécialité dans les départements informatiques des IUT : choix des matériaux linguistiques et didactisation
}

\author{
Arlette Dechet
}

1 En Institut Universitaire Technologique, notre mission première est de préparer nos étudiants à un métier, de les rendre opérationnels sur leur premier emploi et de les aider à rester évolutifs face aux opportunités qu'ils sauront déceler ou qui leur seront proposées.

2 Dans notre domaine d'enseignement de l'anglais nous devons privilégier la formation à la langue de métier : elle assure la crédibilité de l'étudiant à une embauche; elle conforte ses compétences techniques ; elle lui donne de forts points d'appui personnels pour croire en lui et aller, seul, plus loin.

3 Notre dispositif s'articule autour de trois axes d'action : comprendre les besoins de nos étudiants, identifier les besoins du marché, allier ces aspirations qui ne sont pas toujours convergentes en première analyse.

4 Cette situation entraîne des exigences fortes : s'appuyer sur les matériaux linguistiques d'un métier pour permettre l'apprentissage de la langue, transformer ces matériaux linguistiques en situations didactisées.

\section{Le public des IUT}

5 Nos IUT accueillent les étudiants après leur succès baccalauréat (baccalauréats de $\mathrm{A}$ à $\mathrm{H}$ ). La sélection se fait sur dossier, les bacs $C$ et D sont choisis en priorité. L'anglais peut être la première langue soit sept ans d'anglais) ou la deuxième langue (quatre ans d'anglais). 
Le public est donc hétérogène. Le niveau d'anglais s'étale du moyen-faible au très fort. Nous pouvons avoir des étudiants parfaitement bilingues.

De nos jours, un étudiant qui postule à une carrière informatique arrive avec des connaissances techniques sympathiques mais hétéroclites. Parce que des pères Noël successifs lui ont offert quelques matériels et quelques logiciels, parce qu'il a négocié, entre amis, des échanges de programme, parce qu'il a pris plaisir à des relations ludiques entre copains, et qu'il a eu des réussites dans ces différents stades de découverte et d'accomplissement, il s'imagine connaître l'informatique. Ses parents, son entourage l'ont conforté dans cette impression au même titre que les spécialistes à quinze ans (dans les années 1950) du Mécano, (visserie, tôle, engrenages) avaient un destin assuré d'ingénieurs.

7 Il y a éveil à un système. C'est un élément très positif sur lequel nous devons fonder notre dispositif didactique. Il y a méprise entre le ludique et le métier. Nous devons traiter cette interférence positive. Il faut faire passer les étudiants d'une situation passionnante «la bidouille $^{1}$ » à un mode opératif, plus contraignant «la structure», leur donner des normes qui garantissent l'unicité et la reproductibilité de leur approche.

Notre mission en anglais est voisine de celle de nos collègues en informatique. À partir de connaissances hétérogènes, nous nous efforçons de créer une relation structurée à une langue. Notre démarche est modeste: partir de l'existant (niveau des étudiants au départ), fixer les enjeux (ce que désire la profession) et faire réussir chaque étudiant (par structure didactique et accompagnement ludique).

Les étudiants intègrent l'IUT pour deux années (trois trimestres en première année, deux trimestres en deuxième année renforcés par dix semaines de stage en entreprise).

10 L'étude intensive de l'informatique pendant les deux premiers trimestres en IUT va rendre plus homogènes les connaissances et les compétences de l'ensemble de la promotion dans cette discipline.

11 L'anglais exigé est un anglais de métier, nous l'appellerons anglais de l'informatique (nous verrons plus avant en quoi il consiste exactement). Difficile pour un enseignant d'anglais de commencer l'enseignement de l'anglais de l'informatique tant que l'étudiant n'a pas une certaine maîtrise de cette spécificité.

12 Nous ne sommes pas informaticiens, notre rôle n'est pas de faire découvrir et d'expliquer les concepts informatiques (même si nous le faisons ensuite fréquemment). Nous attendrons donc le début du troisième trimestre de la première année pour démarrer cet enseignement de langue de spécialité. Pourquoi le troisième trimestre de la première année ? Pourquoi pas le début de la deuxième année?

$13 \mathrm{Au}$ début du troisième trimestre, les enseignants d'informatique distribuent aux étudiants des documents en anglais (documentation UNIX décrivant et expliquant les commandes de ce système d'exploitation). Ce texte est très technique et pas toujours facile à déchiffrer. Les difficultés sont dues à une certaine lourdeur syntaxique et au lexique souvent jargonneux.

14 Le rôle de l'enseignant d'anglais est d'expliquer les structures ainsi que le sens, l'origine ou la construction des termes inhabituels. L'enseignant d'informatique complète cette première approche en précisant le sens spécifique des concepts techniques.

15 Les efforts conjugués des trois forces (enseignant d'informatique, enseignant d'anglais et étudiant) facilitent la compréhension et la mémorisation de tels documents. Ne parlons 
pas de la motivation des étudiants : elle est évidente puisque ce travail préparé pendant le cours de langue leur permet de s'améliorer dans leur discipline principale.

À la fin du deuxième trimestre de la première année, les étudiants ont abordé le système et l'environnement informatiques ainsi que l'analyse et la conception des systèmes d'information. Ils ont un bagage suffisant pour se plonger dans des textes spécialisés rédigés en anglais.

\section{L'anglais de métier : l'anglais de l'informatique}

17 Quels documents allons-nous choisir? Quel va être l'anglais destiné aux étudiants d'informatique? Nos étudiants se préparent aux métiers de l'informatique. Nos documents doivent répondre à leurs besoins. J'ai conduit une enquête dont l'objectif était précisément d'identifier les besoins actuels en anglais de l'informatique des entreprises et leurs opinions pour 1992. Cette enquête à laquelle une soixantaine d'entreprises prenant des étudiants des départements informatiques en stage ont répondu, est intégrée à ma thèse (Dechet 1990).

Quatre situations sont explorées :

1. Comprendre l'anglais écrit : messages-écrans, notices de matériel, manuels de logiciel, journaux, revues, livres.

2. Rédiger en anglais: messages-écrans, courrier professionnel, résumés ou abstracts, rapports, procédures, traductions, communiquer en temps réel par ordinateur.

3. Comprendre l'anglais oral : conférences, émissions de radio, vidéos professionnelles.

4. S'exprimer oralement en anglais : parler au téléphone, piloter des anglophones, participer à des débats, visiter des salons informatiques.

\section{Les besoins en anglais de l'informatique : résultats de l'enquête}

19 Le premier volet « comprendre l'anglais écrit » fait partie des exigences primordiales de l'entreprise avec un taux qui dépasse $70 \%$ actuellement et augmente de plus de $10 \%$ pour 1992. La demande est particulièrement forte en ce qui concerne la lecture des messages-écrans $(97 \%)$ et la compréhension des manuels de matériels et de logiciels (respectivement $90 \%$ et $93 \%$ ).

Elle est moins forte actuellement pour les autres sources écrites, mais croît fortement en projection sur 1992.

21 Le cas des manuels de matériel et de logiciel est typique. Le plus souvent, il existe une traduction française conformément à la réglementation. Il n'est pas rare que la traduction soit imparfaite ou incomplète et l'informaticien utilisateur est obligé de recourir à la version originale en anglais, pour approfondir un détail de fonctionnement. Nous en avions un exemple concret au département informatique où les enseignants distribuaient une version française d'une documentation UNIX ainsi que la version anglaise. La version anglaise a toujours été plus complète, sa mise à jour plus récente que la traduction française. Cette année, ils ont décidé de ne plus distribuer la version française. 

d'expression écrite en anglais sauf les messages-écrans qui font partie du travail de base. Le besoin d'écrire en anglais existe cependant et l'enquête montre une certaine intensité qui s'accentue à l'horizon 1992. pouvoir suivre des conférences en anglais est très appréciée et le sera encore plus en 1992 avec un taux qui passe de 56 à $79 \%$. La radio est délaissée, car rares sont les programmes radiophoniques consacrés à l'informatique. La vidéo est nettement mieux traitée que la radio et le taux d'exigence à terme fait plus que doubler en moins de trois ans. Pourquoi ? Les constructeurs de matériel et les éditeurs de logiciel utilisent de plus en plus ce moyen d'information pour faire connaître leur produit. Nous devons préparer les étudiants à cette nouvelle forme de communication et d'apprentissage. D'ailleurs, en visitant la bibliothèque du Polytechnic de Brighton en décembre 1991, j'ai remarqué le nombre impressionnant de vidéos ayant trait à l'informatique qui sont mises à la disposition des étudiants. Ces vidéos permettent de s'auto-former ou de compléter l'enseignement universitaire dans les domaines les plus divers tels que la programmation, l'intelligence artificielle, la robotique, la conception assistée par ordinateur, la publication assistée par ordinateur, l'infographie, les réseaux, les télécommunications... Elles expliquent aussi le fonctionnement de produits nouveaux (matériels et logiciels).

24 À Paris j'ai assisté à un certain nombre de conférences dans des expositions et des salons spécialisés en informatique. Une intervention sur deux est faite en anglais chaque fois que le sujet concerne un thème avancé en informatique (l'état de l'art). Avec le Marché unique et l'ouverture des frontières, ce phénomène ne peut que s'amplifier.

Le quatrième volet « s'exprimer oralement en anglais » montre que la demande dépasse rarement les $40 \%$ actuellement, mais double pour 1992. L'aptitude à répondre et à converser au téléphone est appréciée, mais pas encore exigée.

26 Il est plus difficile de se faire une opinion sur les aptitudes à recevoir et piloter des anglophones, à participer à des débats en anglais, à visiter avec profit des salons informatiques à l'étranger. Les taux se situent autour de $40 \%$ à $50 \%$ actuellement pour atteindre $70 \%$ en 1992. Il est clair que les responsables d'entreprise qui ont répondu à l'enquête sont conscients que les informaticiens devront communiquer de plus en plus avec des collègues d'autres pays. Ce n'est pas une urgence, c'est un souci, une attente. Nous devons nous y préparer au plan pédagogique. Le problème de l'enseignement est non seulement de former des étudiants à ce qui est demandé, mais aux nouvelles situations dont nous percevons à travers cette enquête les frémissements.

Le monde de l'informatique était le dernier domaine des croissances exemplaires et des réussites assurées. C'est dans ce monde que, dans les dix dernières années, des réussites techniques et financières ont fait concurrence aux stars de la chanson et de l'écran.

28 Après la phase d'expansion vient celle de maturité. De grandes vedettes de l'informatique disparaissent. D'autres changent de marché. IBM, la référence, licencie. Bull fait alliance. Macintosh ouvre son système. Olivetti s'interroge. Que devient l'avenir professionnel de nos informaticiens?

Le professionnalisme, c'est aujourd'hui de comprendre des messages-écrans, des notices et des modes d'emploi.

Demain, c'est de résumer des revues et des livres informatiques anglais, de rédiger dans cette même langue le courrier professionnel et de visiter des expositions internationales 
pour s'y produire et valoriser son entreprise. Après-demain, c'est négocier de nouveaux marchés, assister à un débat, conduire un débat, rédiger rapports et procédures en anglais.

\section{Les matériaux linguistiques et leur didactisation}

31 Pour obtenir ce professionnalisme, nous disposons de nombreux matériaux linguistiques. Lesquels choisir et comment les didactiser pour animer nos cours d'anglais?

\section{Matériaux linguistiques de première nécessité : les messages- écrans}

Les messages-écrans apportent une aide conviviale au pilotage de la machine, de son système ou du logiciel d'exploitation. Ces messages guident l'opérateur dans le choix des commandes d'entrée dans le réseau, dans la sélection d'une fonction ou dans le mode de présentation d'un résultat.

Notre Institut Universitaire a acquis l'un des derniers nés d'un gros système IBM, l'IBM 9370, modèle 90. Les messages-écrans sont en anglais. Nous pouvons soit obtenir un listage de tous les messages-écrans proposés par le système, soit travailler directement avec les étudiants sur les terminaux IBM.

Le listage des messages-écrans est d'un coût acceptable, mais il est difficile d'animer un travail pédagogique à partir des messages listés sur papier. La mobilisation d'heures de gros système ne peut être trop longue : les étudiants ont besoin des terminaux pour leurs travaux informatiques.

Nous avons constaté que la syntaxe des messages-écrans est semblable, quels que soient le type et la puissance du système informatique. Les verbes, les expressions sont souvent identiques; seuls changent quelques termes spécifiques à un système; leur nombre est restreint. Nous utilisons donc les messages-écrans d'un logiciel de traitement de texte anglais WORD4, en faisant travailler les étudiants sur des tâches identiques ou différentes. Dechet (1990) présente un cours complet conçu sur WORD4 pour étudiants informaticiens. J'ai rédigé dans Le Bulletin de l'EPI (Dechet 1991) un article sur toutes les activités didactiques qui pouvaient être créées sous traitement de texte. Je ne m'attarderai donc pas sur cette démarche didactique.

Rappelons cependant que tout en se familiarisant avec un outil que peu d'entre eux maitrisent, le traitement de texte, les étudiants apprennent sans s'en rendre compte les termes anglais qui permettent de comprendre son fonctionnement. Ils se retrouvent dans leur élément (le monde des machines) et en situation de jeu. Cette démarche ludique est efficace et très appréciée.

\section{Les pages de manuels}

37 Je mets à leur disposition le manuel utilisateur en anglais. À eux de trouver l'information dont ils ont besoin pour comprendre le rôle d'une instruction et son fonctionnement. Ils cherchent, trouvent, déchiffrent, déduisent et s'approprient les informations. L'enseignant les aide. Il leur explique pourquoi une structure syntaxique leur a semblé 
difficile, il leur donne le sens de certains mots encore inhabituels à ce stade de leur formation.

J'ai mentionné un peu plus haut la documentation UNIX que les enseignants d'informatique nous soumettaient.

Il est toujours difficile de travailler sur une telle documentation. Nous ne sommes pas sur machine, comme dans le cas précédent, les étudiants feuilletant une documentation et utilisant, sur le champ, l'information trouvée pour obtenir un résultat sur machine. Cette documentation (d'une quarantaine de pages) est un ensemble d'instructions sur papier dont la lecture n'a rien divertissant. Les étudiants se lassent très vite, nous aussi.

Nous proposons aux étudiants le travail suivant : lecture de toute la documentation chez soi. Étude approfondie de deux à trois pages par étudiant: numéroter les lignes du document, surligner les difficultés syntaxiques ou lexicales, les traduire ou donner une explication en français ou en anglais.

Ce passage par la traduction peut choquer certains collègues. Dans toute notre formation de professeur de langue étrangère, on nous a enjoints, en effet, de ne pas passer par la traduction pour travailler la compréhension.

2 Que penser de ces consignes? Sont-elles encore valables quand il s'agit de langue de spécialité ? Nous devons rendre nos étudiants opérationnels au plus vite. Pourquoi leur faire perdre un temps précieux à trouver une explication en anglais qui risque d'être approximative alors que la traduction les oblige à plus de précision ? Le but de ce travail n'est pas d'acquérir un maximum de vocabulaire et de structures de communication, mais de comprendre un texte pour pouvoir mieux tirer profit d'une machine, d'un logiciel ou d'un langage informatique. Soyons rapides et efficaces.

L'obligation de travailler sur des documentations techniques aussi ingrates ne nous facilite pas la tâche. Les cours risquent d'être soporifiques. J'utilise donc la stratégie de Michel Perrin, Professeur d'anglais à l'Université de Bordeaux 2.

1. Panoplie d'activités : multiplication des modes d'animation.

2. Découpage des activités : durée déterminée pour chaque thème ou mode d'intervention.

3. Recentrage : pour une meilleure fixation des nouvelles données linguistiques.

4 Ces activités d'animation, de réanimation et de fixation peuvent être des exercices d'assortiment : il faut assortir des expressions et leur définition, des expressions et leur traduction, des exercices lacunaires, des exercices de remise en ordre, bref, toute activité brève qui peut se présenter sous forme de jeu, quizz et puzzle (voir plus loin activités didactiques).

Ces activités peuvent annoncer le vocabulaire ou la syntaxe qui va suivre ou rappeler ce qui a déjà été vu. Elles éveillent la curiosité de l'étudiant en lui donnant l'envie d'en savoir plus. Elles permettent de fixer certaines expressions. Elles brisent le rythme, rompent la monotonie d'un cours en rendant actifs ceux qui auraient tendance à se laisser bercer par le travail des autres.

Je les appelle exercices-trampoline (trampoline-exercises). Pourquoi cette dénomination? Un trampoline est un lieu de jeu, de bonds, de rebonds, de galipettes, de sauts (parfois périlleux), de récurrences, de passages obligés... Et une langue ? Un trampoline mobilise la vivacité, la souplesse, l'agilité, la légèreté, l'adaptabilité. Un trampoline favorise la drôlerie, la clownerie, la pitrerie, mais tout autant la précision, la réponse instantanée et 
la vivacité. L'efficacité naît de ce curieux mélange de ludisme et de coercition. N'en est-il pas de même de l'apprentissage d'une langue?

47 À l'instar de Michel Perrin, j'introduis ces exercices-trampoline dans toute approche pédagogique à intervalles réguliers. Les mots et les structures qui bondissent et rebondissent sont saisis au vol par les étudiants réveillés et amusés.

48 Au-delà des jeux et des situations ludiques qui déconnectent les étudiants de leurs habitudes routinières et leur donnent la force de conquérir de nouveaux espaces langagiers, nous devons leur donner accès à un horizon linguistique plus ouvert.

\section{Matériaux linguistiques complémentaires}

49 Reprenons les quatre types de documents informatiques identifiés par A. Pradeilles (1981) pour un même fait scientifique : la communication scientifique entre spécialistes, la lettre d'information (newsletter); le manuel pédagogique scolaire ou universitaire; la revue scientifique vulgarisation; la presse à grande diffusion pour grand public.

50 Voyons aussi la pyramide lexicale de langue de spécialité de Michel Perrin (1990) qui rend compte du lexique que l'on trouve dans chaque type de document, en commençant par le haut de la pyramide.

51 - Les «topic-specific terms » sont des termes très spécifiques qui sont propres à une science ou à une technique. Le spécialiste les reconnaît immédiatement.

52 - Les «subject-specific terms » sont des termes spécifiques qui peuvent être utilisés dans plusieurs contextes scientifiques et techniques.

53 - Le «general scientific language » est le langage caractéristique du discours scientifique et technique : vocabulaire et syntaxe compris.

54 - Le «specific use of general language» est le langage de tous les jours utilisé à des fins scientifiques et techniques.

55 - Le «foundation language » est le langage de tous les jours qui permet de communiquer dans quelque discours que ce soit. Sont incluses dans cette strate toutes les structures grammaticales.

56 L'inventaire d'A. Pradeilles correspond aux documents écrits qui couvrent un même fait scientifique. On peut le plaquer sur la pyramide de M. Perrin. La presse à grande diffusion pour grand public utilisant le plus de «foundation language » alors que la communication entre spécialistes se contente d'un minimum de «foundation language» (il en faut un minimum pour structurer le discours) et d'un maximum de "topic-specific terms » pour décrire avec précision le fait scientifique.

57 Pour que l'inventaire des documents écrits soit complet, ajoutons : les messages-écrans, les manuels de fabricants de matériel et de logiciel (j’ai déjà indiqué comment les choisir et suggéré comment les didactiser), les publicités: elles sont souvent riches en vocabulaire de spécialité, les livres ayant trait à l'informatique, les dictionnaires unilingues et bilingues de spécialité et pour les documents oraux : les cassettes audio (il y en a peu), les cassettes vidéo : elles sont déjà nombreuses.

58 Revenons aux documents qui couvrent un même fait scientifique. Un texte tiré de la presse à grande diffusion sera-t-il plus facile à déchiffrer qu'une communication scientifique entre spécialistes? Rien de moins évident. 

avancée pour faire progresser la Connaissance. Elle est prématurée pour des étudiants d'IUT qui limitent leurs études à Bac +2 et éprouvent déjà des difficultés à interpréter des textes abstraits ou théoriques en français. Ce constat ne doit pas interdire à l'enseignant de proposer des travaux sur des textes difficiles qui améliorent les capacités d'abstraction et de spéculation des étudiants et leur donnent le goût de s'intéresser à des écrits de spécialité et le désir de se dépasser (encore faut-il se les procurer!). La situation est délicate; elle doit être gérée avec un charisme pédagogique certain. Les exercices trampoline seront d'un très grand secours dans ces moments-là.

\section{La lettre d'information}

62 J'ai ajouté dans l'inventaire d'A. Pradeilles, la lettre d'information (Newsletter). La lettre d'information annonce et décrit des produits nouveaux. Elle est différente de la communication scientifique. Elle est moins abstraite. C'est un document conçu par des spécialistes pour d'autres spécialistes. Ceux de la production s'adressent à ceux de la commercialisation.

63 Ces documents sont réservés aux professionnels et ne doivent pas être communiqués au grand public. La lettre d'information s'intéresse à de multiples domaines de l'informatique (en particulier, aux différents types de logiciels et de matériels). Ces documents paraissent en général tous les mois. Ils pourraient présenter un intérêt pour notre enseignement, mais leur caractère trop spécifique, leurs descriptions trop détaillées dépassent nos besoins et leur coût (ils sont élevés), nos budgets.

Nous désirons mettre en permanence à disposition des étudiants des documents qui ont valeur de référence pour aborder un sujet ou éclaircir un concept de base de l'anglais de l'informatique. Ces documents de référence se trouvent dans les manuels pédagogiques scolaires ou universitaires.

\section{Le manuel pédagogique scolaire ou universitaire}

Il faut distinguer deux types de manuels: celui rédigé en anglais et utilisé par les étudiants d'informatique d'Outre-Manche ou d'Outre-Atlantique et celui conçu par des enseignants d'anglais, pour des informaticiens. 


\section{Le manuel d'informatique}

66 J'en utilise plusieurs. Ils sont d'excellente qualité et présentent l'environnement informatique de façon claire et structurée. Ils nous rendent compte des différents thèmes liés à l'informatique. Ils servent d'ouvrages de référence qui me permettent de mieux choisir des articles dans les revues ou magazines d'informatique.

Computing Science de Peter Bishop (1984) prépare au Royaume-Uni à l'obtention du A level. Il donne une vision complète du monde informatique. Il se termine par un glossaire et un index.

Computer Science 'A Modern Introduction' de Les Goldschlager and Andrew Lister (1988) est conçu pour les étudiants des Polytechnics britanniques. Il traite des ordinateurs, des logiciels, de programmation, de la théorie des algorithmes et de la réalisation d'algorithmes. Il contient un index.

Computers and Data Processing de Steven L. Mandell (1985) s'adresse aux étudiants d'université des États-Unis. Il se divise en cinq parties : le traitement de l'information, la technologie, la programmation, les systèmes, et les ordinateurs dans la société. Il est abondamment illustré et il est complété par un glossaire assez riche et un index.

Introduction to Computers and Information Systems with BASIC de Thomas H. Athey and Robert W. Zmud (1988) est destiné aux étudiants informaticiens ou non informaticiens. Son objectif est d'expliquer comment fonctionnent les ordinateurs et dans quelle mesure ils simplifient et améliorent la vie de tous les jours.

71 Schémas et photos illustrent les différents chapitres qui traitent en profondeur de la société informatisée, des machines, des langages, des logiciels, de la programmation, des systèmes de gestion de l'information et des métiers de l'informatique. Chaque chapitre se termine par un résumé et des questions de révision. Un glossaire reprend les termes qui ont été définis dans l'ouvrage. Les mots clés sont repris en index.

Tous les ouvrages sont régulièrement mis à jour. Les concepts sont explicités avec clarté et simplicité. Des tableaux synoptiques reprennent les idées principales. Ce sont des outils de travail et de référence pour tout étudiant anglophone et de précieux auxiliaires pour l'enseignement ou l'apprentissage de la langue de l'informatique car leur contenu correspond exactement à ce que nos étudiants doivent connaître s'ils veulent pouvoir communiquer en anglais dans leur métier

73 Au cours de chaque voyage au Royaume-Uni ou aux États-Unis, je cherche à découvrir de nouveaux manuels pour les comparer à ceux que je viens de citer.

Ces manuels sont conçus pour l'apprentissage de la technologie par des anglophones et non pour l'assimilation de la langue par des non-anglophones. Par conséquent, aucun exercice linguistique n'est proposé. L'enseignant y trouve seulement une source d'animation (un corpus de textes spécialisés) qu'il doit rendre didactique. Le travail est intéressant, mais long et parfois déroutant. Les activités que nous proposons un peu plus avant dans activités didactiques peuvent toutes être utilisées pour la didactisation, mais il est souvent plus commode d'utiliser des manuels spécialement rédigés pour l'enseignement de l'anglais de l'informatique. 


\section{Le manuel d'anglais de l'informatique}

75 La plupart des manuels actuellement commercialisés sont publiés par des maisons d'édition anglaises ou américaines. Peu sont proposés par des éditeurs français.

\section{Les manuels anglo-saxons}

English for Computer Science de Norma D. Mullen et P. Charles Brown (1987) s'adresse à des étudiants ayant un niveau moyen en anglais. Les exercices proposent différentes techniques de lecture et de compréhension de textes informatiques. Certains points de grammaire sont explicités. Un bref glossaire complète le manuel. Un corrigé et des cassettes sont vendus séparément.

English in Computing de Eric H. Glendinning and John McEwan (1987) convient à des débutants. Les concepts de base de l'informatique sont présentés sous forme d'exercices variés qui privilégient tour à tour la compréhension écrite et orale et l'expression écrite et orale. Un glossaire et un corrigé des exercices se trouvent en fin de livre. Des cassettes accompagnent le manuel.

Computer Science de Richard A. Meyers (1984) propose à des étudiants de niveau débutantmoyen des dialogues, l'explication de mots-clés et de courts exercices de vérification des connaissances. Les mots-clés sont regroupés dans un glossaire à la fin du livre avec le corrigé des exercices. Une cassette est vendue séparément.

La série Special English chez Cassell comprend plusieurs manuels consacrés à l'informatique. Chaque manuel s'adresse à des étudiants de niveau débutant ou moyen.

Chaque unité commence par un dialogue suivi de quelques exercices qui facilitent l'acquisition de structures et du vocabulaire nouveaux. Ils peuvent être faits oralement ou par écrit. L'étudiant est ensuite invité à lire un texte qui correspond au thème de l'unité et à répondre aux questions de compréhension. Le corrigé des exercices est donné en fin de manuel avec un glossaire. Des cassettes complètent la collection.

81 Les différents titres de cette série sont : Computers de Edward Humby and Philip Robinson (1981), Computer Programming de Philip Robinson (1981), Computer Applications de Edward Humby and Philip Robinson (1980).

\section{Les manuels français}

En France, cinq manuels ont été publiés. L'anglais de l'informatique s'enseigne de plus en plus. Nul doute que d'autres ouvrages seront publiés dans les années à venir.

This Quiet Revolution, de Y. Leray, A. Pradeilles et N. Vigouroux-Frey (1980). Cet ouvrage est un recueil de textes de langue anglaise sur l'informatique, ses applications et leurs conséquences dans le monde. Ce livre, malheureusement épuisé, est une mine d'informations directement utilisables par l'enseignant à condition de faire l'effort de construire un cours à partir de chacun des thèmes proposés.

84 L'anglais pour informaticien de Liliane Gallet (1981) est un manuel d'initiation à l'anglais de l'informatique. Il se compose d'une grammaire et d'un ensemble de textes de spécialité. La grammaire permet l'apprentissage ou l'approfondissement de points de grammaire spécifiques, les textes entraînent à la lecture de brochures techniques d'informatique. Des fiches de lecture, des questions et des tests accompagnent chaque texte. Un glossaire de 500 mots usuels et un index renvoyant aux paragraphes de grammaire se trouvent à la fin du manuel. 

domaines liés à l'informatique. Du matériel aux logiciels, des réseaux locaux aux transmissions par satellite, de l'intelligence artificielle aux virus... Chaque unité se divise en six parties. Chacune développe l'aptitude à écouter, lire, comprendre et rédiger un texte informatique. De nombreuses expressions usuelles et idiomes propres à la langue informatique sont traduits. Un point de grammaire suivi d'exercices est associé à chaque unité. Des activités variées sous forme de simulations de situations professionnelles sont proposées pour inciter l'étudiant à communiquer. Un index en fin de manuel permet de naviguer facilement dans l'ouvrage. Un livre de corrigés et trois cassettes complètent cet ensemble de cours. GOTO s'adresse à tous les niveaux, mais il est un peu trop complexe pour les débutants.

English For Information Technology de Michael Brookes et François Lagoutte (1989) comprend huit sections traitant des différents domaines de l'informatique. Ce manuel est accompagné de deux cassettes et d'un livre de corrigés. L'ensemble est conçu pour les étudiants et les professionnels qui se spécialisent en informatique.

L'anglais pour l'informatique de Liliane Gallet et Jean Brossard (1990) se compose d'articles de presse, de brochures techniques et de textes spécifiques qui font chacun, l'objet d'une fiche de lecture. Des exercices renvoient à un précis grammatical inclus dans l'ouvrage. Le dernier chapitre comprend une liste de faux amis, les verbes irréguliers, un lexique et un index-glossaire.

Les articles de tous ces manuels sont tirés de revues scientifiques, de magazines à grande diffusion ou de livres d'informatique. La didactisation est préparée. Cela rend la tâche plus facile aux enseignants. Mais l'informatique est en perpétuelle évolution et le contenu de ces manuels peut très vite devenir obsolète.

89 De plus, pour son efficacité, une activité doit correspondre à la personnalité d'un enseignant et au profil de ses étudiants. Une activité réussie avec un groupe et un enseignant peut être un fiasco total dans d'autres circonstances. Il est évident que selon le niveau d'anglais des étudiants, le contenu linguistique et le type d'activité seront différents.

90 Si l'enseignant à peu de temps pour concevoir ses cours ou s'il a une maîtrise limitée de l'anglais de l'informatique, nous lui conseillons ces manuels. Ils peuvent aussi servir d'ouvrages de référence pour lui, et pour les étudiants. Les enseignants pourront ensuite puiser directement dans les revues, magazines ou livres des matériaux linguistiques plus récents qui viendront compléter ou remplacer les textes des manuels.

91 Faisons l'inventaire de ces documents (inventaire non exhaustif, mais suffisant pour notre propos) et voyons ensuite comment les didactiser.

\section{La revue scientifique de vulgarisation}

Voici quelques titres des revues anglo-saxonnes auxquelles mon institut ou moi-même sommes abonnés: Computerworld, hebdomadaire américain ; Byte, mensuel américain; Mini-Micro Systems, mensuel américain; PC Magazine, mensuel américain; Personal Computer World, bimensuel britannique ; Computer Weekly, hebdomadaire britannique.

Les articles de Personal Computer World sont les plus clairs, ensuite viennent PC Magazine, Mini-Micro Systems. 

solution immédiate au problème qu'ils cherchent à résoudre. Il appartient à l'enseignant
de langue de leur donner ou de ranimer le goût, le plaisir de s'investir dans la lecture et
de leur faire comprendre qu'il ne peut y avoir haute compétence professionnelle sans
retour fréquent aux sources : les livres. 
104 Il en existe d'excellents sur l'intelligence artificielle, les robots, les satellites... Nous devons les leur faire connaitre, mais n'aurons vraisemblablement pas le temps de les utiliser pendant les cours, sauf pour des projets de synthèse.

\section{Les publicités}

105 Il est facile de collectionner de multiples publicités lors de la visite d'un salon informatique en France ou à l'étranger. Cette collecte peut être faite par l'enseignant ou par les étudiants. Certains fabricants rédigent leurs publicités en anglais et en français : profitons de leur traduction.

106 Dans les magazines de vulgarisation, la publicité est abondante. C'est un matériau exceptionnel pour agrémenter nos cours de documents pertinents, cohérents, originaux et parfois très drôles².

107 La publicité que l'on trouve dans les salons ou les magazines est en prise directe sur la nouveauté. Elle donne l'occasion d'étudier la puissance de contraction de l'anglais qui est portée à son maximum dans les messages publicitaires. Elle permet de se familiariser avec une écriture très elliptique, proche des formes condensées des messages-écrans.

\section{Les dictionnaires}

108 L'utilisation des dictionnaires comme outil de décodage ne pose aucun problème. Un dictionnaire sécurise, il rassure. Les étudiants apprécient les dictionnaires spécialisés qu'ils estiment efficaces et de consultation facile. Ils se servent de lexiques présentés sous forme de logiciel (les dictionnaires électroniques) qui permet une recherche quasi instantanée.

109 Comme pour le livre, l'enseignant de langue doit réagir contre la propension des étudiants à se contenter d'un résultat immédiat, limité au simple décodage d'un mot ou d'une expression. Il faut les initier au plaisir d'explorer dans un dictionnaire toutes les variétés sémantiques d'un mot, de se former à ce mot, à son contexte, à ses arborescences, à tous les avatars de sa carrière de mot. En prenant le temps de s'en imprégner, l'étudiant dépasse le niveau de la tâche pour améliorer sa culture.

110 Les documents à notre disposition pour la conception de nos cours sont nombreux, riches et variés. Nous privilégions les plus clairs et les plus structurés. Voyons sous quelle forme les présenter et les transmettre.

\section{Didactisation, activités didactiques}

111 Les activités didactiques sont composées des différents exercices, animations ou travaux que l'enseignant propose aux étudiants afin qu'ils améliorent leurs connaissances en langue étrangère. Ces activités sont conçues en fonction des besoins langagiers des étudiants.

112 L'enquête sur les besoins en anglais de l'informatique a révélé que le futur informaticien doit être capable de déchiffrer des documents informatiques en anglais, quel que soit le type d'entreprise dans laquelle il sera accueilli. Il est souhaitable, bien que pour le moment non indispensable, qu'il sache communiquer avec des professionnels anglosaxons. 
113 Avec 1992 et l'ouverture du marché européen, il sera urgent que les futurs informaticiens français puissent participer aux échanges internationaux qui se font en anglais, s'ils ne veulent pas être éliminés de certains marchés ou prendre du retard dans l'apprentissage des techniques nouvelles. Quelles activités didactiques leur proposer?

114 Dressons la liste de celles dont l'enseignant dispose lorsqu'il conçoit des cours d'anglais informatique. Si l'on pense en terme de communication, il est difficile de séparer les activités portant sur la compréhension et la production langagières. Je me trouve cependant dans l'obligation de dissocier ces deux composantes d'un même continuum pour des raisons de clarté.

115 L'originalité et l'efficacité d'un cours dépendront du choix et du mélange judicieux de ces activités ainsi que de la pertinence et de la clarté de leur contenu linguistique.

\section{Compréhension des documents}

116 Un document peut être abordé de différentes façons: l'approche peut être globale, sélective ou détaillée.

117 L'enseignant peut combiner ces approches ou se limiter à celles qui lui semblent les plus pertinentes par rapport aux objectifs didactiques qu'il s'est fixés.

\section{Approche globale}

118 Survol : le texte est parcouru très vite pour savoir de quoi il s'agit, quel est son intérêt et quelle est sa structure. On examine le plan du texte en s'aidant de la mise en page, de la typographie et du graphisme. On sélectionne les passages à examiner plus à fond. On devine le sens des mots inconnus par inférence.

\section{Approche sélective}

119 Repérage : le texte est parcouru pour y trouver une information particulière. Écrémage : Le texte est parcouru pour repérer les idées importantes ou nouvelles. On recherche les mots-clés, le sens des titres et des sous-titres. On essaie de comprendre la légende de certaines illustrations et de les interpréter.

\section{Approche détaillée}

On étudie un texte dans le détail en examinant certains passages plus avant (approfondissement) ou en s'obligeant à les reformuler (reformulation).

\section{Approfondissement}

On recherche les relations dans une phrase puis les relations entre les phrases (articulation logique) en s'intéressant à la cohésion grammaticale et à la cohésion lexicale. On comprend les concepts et on identifie la nature de leurs enchaînements. On trouve l'idée principale d'un paragraphe, puis les idées secondaires ou les détails de l'idée principale. On s'assure de la cohérence de l'ensemble.

Quel que soit le type d'approche utilisé, l'étudiant est invité à prendre des notes. Les notes facilitent la mémorisation des informations nouvelles. Les notes utilisées ultérieurement pour des travaux de reformulation permettent une meilleure intégration de ce qui est nouvellement acquis. 


\section{Reformulation}

On recompose le texte dans son langage pour se l'approprier ou pour le transmettre à d'autres. Cette activité peut prendre différentes formes: reformuler les passages difficiles; contracter le texte pour en faire un résumé ; condenser le texte en un schéma explicatif ou un tableau récapitulatif; composer un texte à partir de dialogues; rédiger un texte à partir d'un résumé, d'images, d'une publicité, de schémas ou de tableaux; réécrire un texte en changeant de niveau de langue.

Des questions de différente nature peuvent être posées pour préparer, aider ou compléter la lecture de documents tout en facilitant l'appropriation et l'intégration des connaissances nouvelles.

\section{Questions}

Les questions de compréhension sur un énoncé peuvent être présentées de deux manières : aucune réponse n'est donnée ; un choix de réponses est proposé.

\section{Questions sans proposition de réponse}

Questions fermées : il n'y a qu'une réponse possible. Ce sont souvent des questions où l'on répond par oui ou par non. Questions ouvertes : elles sont introduites par les pronoms interrogatifs ou les conjonctions "Qui, que, quoi, où, comment, pourquoi...». Les réponses peuvent être variées et de tailles plus ou moins longues.

\section{Questions avec choix de réponses}

127 Deux réponses sont proposées. L'étudiant porte un jugement de vérité : vrai - faux. Plus de deux réponses sont suggérées (choix multiple). L'étudiant doit choisir parmi toutes ces options (voir ci-dessous la variété des exercices de choix multiple).

\section{Production écrite : exercices}

Les questions qui permettent de vérifier la compréhension d'un passage peuvent être remplacées par différents types d'exercices. Ces mêmes exercices peuvent être conçus pour une meilleure connaissance du lexique et de la grammaire ou pour l'évaluation.

\section{Exercices de sélection (choix multiple)}

Le choix multiple peut se faire entre 4 ou 5 éléments. Les éléments peuvent être des mots, des expressions, des phrases ou des paragraphes.

Deux possibilités : dans les éléments proposés, il y a obligatoirement l'élément à trouver ; dans les éléments proposés, il n'y a pas obligatoirement l'élément à trouver, il est remplacé par une expression du type « None of the above».

\section{Exercices lacunaires (à trous)}

131 Les trous peuvent être faits de manière aléatoire ou non. Dans le premier cas, on parle de tests de closure (cloze tests). Les tests de closure ont été inventés par Wilson L. Taylor (1953). Un mot sur cinq est supprimé. Par mot on entend un ensemble séparé des autres par un espace blanc. 
Ces exercices mesurent plutôt la compréhension structurale que la compréhension lexicale. Parfois ce n'est pas un mot sur cinq qui est supprimé, mais un mot sur six ou sept ou huit, etc.

Les exercices lacunaires peuvent se présenter de deux façons. Avec aide, on sélectionne l'élément manquant à partir d'un ensemble d'éléments qui sont proposés en amont ou en aval de l'exercice. Il peut y avoir autant d'éléments qu'il y a de trous ou plus d'éléments qu'il y a de trous. Sans aide, on doit deviner l'élément manquant.

\section{Exercices de remise en ordre}

On remet dans l'ordre : des caractères à l'intérieur d'un mot (anagramme), des éléments à l'intérieur d'une phrase, des éléments à l'intérieur de plusieurs phrases, des phrases à l'intérieur d'un paragraphe, des phrases à l'intérieur de différents paragraphes, des paragraphes entre eux.

\section{Exercices d'assortiment}

Il faut assortir: des passages avec des titres, des passages avec des illustrations, des passages français avec des passages anglais (ou vice versa), des mots avec des définitions ou explications, des détails avec des idées principales, des idées principales avec des paragraphes, des passages avec des résumés, des segments de phrases entre eux (pour qu'ils forment une phrase cohérente).

\section{Exercices de transformation}

Il s'agit de changer la structure syntaxique d'une ou de plusieurs phrases sans en modifier la signification. La modification a lieu sur l'axe syntagmatique. On peut aussi avoir à regrouper deux ou plusieurs phrases entre elles.

\section{Exercices de substitution}

On garde la structure syntaxique d'une phrase donnée, mais on change certains éléments de la phrase, ce qui en modifie le sens. La substitution s'y opère sur l'axe paradigmatique.

\section{Exercices de recherche d'erreurs}

L'étudiant trouve les erreurs qui ont été volontairement insérées dans un texte. Il les explique et les corrige. Ces erreurs peuvent être syntaxiques, morphosyntaxiques ou lexicales. Elles peuvent porter sur une ponctuation volontairement incongrue ou inexistante.

\section{Exercices de description}

Les étudiants décrivent ce qu'ils voient sur des photos, des illustrations, des schémas ou des graphiques.

\section{Exercices de reformulation}

L'exercice de reformulation peut être fait à partir d'énoncés discursifs ou à partir d'éléments isolés. On joue avec le mot et ses différentes acceptions ou avec la phrase et le paragraphe, on transforme le mode d'expression.

Avec le mot: l'étudiant trouve des synonymes, des antonymes, des homonymes. Il peut aussi chercher les hétéronymes (mots de racine différente, mais formant ensemble une structure sémantique [Dubois 1973]), les superordonnés ou hyperonymes (les termes dont le sens inclut le sens ou les sens d'un ou de plusieurs autres termes, appelés alors hyponymes [ibid.]). Ces deux derniers types d'exercices préparent à un travail sur la classification. Il trouve les dérivés d'un mot. 
143 L'enseignant peut proposer la définition d'un mot et l'étudiant trouve le mot à partir de cette définition ou vice versa. Il suggère une liste de mots dans laquelle un mot ne peut être associé à l'ensemble des mots. Il s'agit de le trouver et d'expliquer pourquoi il n'a rien à faire dans cette liste.

Du mot à la phrase : l'enseignant propose une liste de mots à partir desquels l'étudiant composera un texte. Cette liste peut être plus ou moins longue. La contrainte peut être plus ou moins grande : tous les mots doivent être utilisés ou uniquement certains d'entre eux.

Avec la phrase: L'enseignant soumet aux étudiants quelques phrases [des proverbes, slogans et aphorismes] et leur demande de changer quelques mots à l'intérieur de ces phrases pour qu'il y ait détournement de sens.

146 Les phrases modifiées doivent rester cohérentes et si possible garder la mélodie qu'elles avaient avant la substitution de certains mots par d'autres. C'est Marina Yaguello (1981 : 113) qui m'a inspiré cet exercice. L'exemple qu'elle donne est : " Take care of the pence and the pounds will take care of themselves » qui devient "Take care of the sense and the sounds will take care of themselves $"$.

147 Voici un autre exemple de détournement de sens trouvé dans une publicité sur les virus ( PC magazine) : a hare raising experience pour a hair-raising experience ${ }^{3}$ (allusion aux virus qui se multiplient comme des lapins, ici des lièvres). La difficulté d'en venir à bout vous fait dresser les cheveux sur la tête.

148 Jouer, faites jouer. Prenez plaisir à transformer votre étudiant en meneur de jeu.

149 De la phrase au paragraphe: l'étudiant simplifie la syntaxe d'une phrase trop compliquée. Il réduit la taille d'un paragraphe en ne gardant que l'essentiel. Il réduit la taille de tout un énoncé. La réduction peut varier selon l'objectif recherché (de $10 \%$ jusqu'à $90 \%$ ).

150 Transformation du mode d'expression : à partir d'un dialogue, il écrit un texte (ou vice versa) ; à partir d'un texte, il rédige un ensemble d'instructions (ou vice versa); à partir d'une publicité, il écrit un article (ou vice versa) ; à partir d'un rapport, il rédige une lettre (ou vice versa).

\section{Exercices de traduction}

151 La traduction de la langue étrangère à la langue maternelle ou de la langue maternelle à la langue étrangère permet de comparer les deux systèmes linguistiques.

Elle fait prendre conscience à l'apprenant du fonctionnement d'une langue par rapport à une autre et de la valeur exacte de certaines entités linguistiques. Ne pas connaître le sens exact d'un terme technique peut avoir des répercussions désastreuses.

Divisons les exercices de traduction en deux catégories: ceux qui ont pour but de contrôler si certains points de grammaire sont bien assimilés et ceux qui permettent de restituer le discours dans une autre langue sans erreur sémantique.

1- La traduction pour vérifier que les règles de grammaire sont bien assimilées : le thème permet de mettre en application la règle de grammaire que l'on vient d'apprendre; la version permet de retrouver la règle de grammaire à partir d'exemples variés. 
2- La traduction pour restituer un discours dans une autre langue : - version et thème : Il est difficile de restituer un texte dans une autre langue en gardant la précision sémantique. Les textes techniques sont cependant plus faciles à traduire que les textes littéraires. La plupart des concepts techniques n'ayant qu'une traduction possible parce qu'un seul sens bien défini. La difficulté vient de ce que nombre de concepts ou termes nouveaux créés dans les pays de langue anglaise n'ont pas encore été traduits en français. Leur traduction dans le Journal officiel arrive souvent quelques mois (voire quelques années) après leur création et leur utilisation.

Je propose aux étudiants certains procédés de traduction (Vinay \& Darbelnet 1977 : 47-55) : l'emprunt : une unité linguistique d'une langue est utilisée et intégrée dans une autre langue; le calque : «on emprunte à la langue étrangère le syntagme, mais on traduit littéralement les éléments qui le composent» (Vinay \& Darbelnet 1977 : 47) ; la traduction littérale : la traduction d'une langue à l'autre peut se faire mot à mot; la transposition : on fait appel à d'autres catégories grammaticales pour traduire un mot ou une expression; la modulation : le point de vue est différent d'une langue à l'autre ; l'équivalence : le message tout entier sera traduit par une expression équivalente (les proverbes, les expressions idiomatiques...); l'adaptation: «la situation à laquelle le message se réfère n'existe pas dans la langue d'arrivée, et doit être créée par rapport à une autre situation, que l'on juge équivalente. C'est donc ici un cas particulier de l'équivalence, une équivalence de situations » (Vinay \& Darbelnet 1977 : 52).

157 Je rappelle aux étudiants qu'une traduction ne doit jamais être ambiguë et que tous les traits pertinents doivent être restitués. Je leur apprends à se servir des dictionnaires unilingues et à ne pas abuser des dictionnaires bilingues.

\section{Production orale}

Les exercices proposés précédemment peuvent rebondir sur des activités orales. Si ces activités sont enregistrées sur cassette, le texte sera lu plus ou moins rapidement au moment de l'enregistrement pour faire varier le niveau de difficulté. Pour la même raison, les pauses qui permettent quelques secondes supplémentaires de réflexion avant de donner sa réponse seront plus ou moins longues.

Devons-nous proposer des exercices de phonétique ? Nos étudiants sont censés avoir des connaissances de base en phonétique. Nous avons constaté que des cours systématiques de phonétique ne les aidaient pas à maîtriser les sons, mais bien au contraire les perturbaient (ils mélangent tout). Il est cependant intéressant de leur rappeler sous forme de tableau synoptique ce que sont les symboles phonétiques.

Ils pourront ainsi retrouver comment se prononce un mot nouveau en consultant un dictionnaire. Il est bon d'insister sur l'opposition entre certaines prononciations de voyelles et de diphtongues inhabituelles ou inattendues ainsi que sur certaines accentuations. Cela peut se faire sous forme d'exercices-trampoline.

Si besoin est, il ne faut pas hésiter à rappeler certaines règles d'intonation, en s'arrangeant, pour que ces règles, bien que décrites, soient plutôt assimilées par mimétisme.

Fin 1992 approche. Les Européens vont se déplacer. De plus en plus de Français iront traiter des affaires au Royaume-Uni, de plus en plus de citoyens britanniques visiteront 
des entreprises françaises. La langue véhiculaire de la Communauté européenne sera l'anglais, il faut donc absolument pouvoir communiquer en anglais.

Les activités proposées ensuite sont conçues pour améliorer directement la capacité à communiquer. Elles viennent en complément des activités précédentes.

\section{Jeux de rôles et simulations}

Lorsqu'on parle de jeux de rôles et de simulations, on pense souvent qu'il s'agit d'activités identiques. Il est vrai qu'elles se ressemblent dans la plupart des cas. Elles présentent cependant de réelles différences. Dans une simulation, l'enseignant propose aux étudiants de simuler une situation inspirée de la réalité. Ils peuvent jouer le rôle qui correspond à leur personnalité ou un rôle plus ou moins éloigné de leur personnalité.

un jeu de rôles, l'étudiant doit incarner un rôle qui n'est pas le sien dans la vie quotidienne. Dans une simulation l'accent est mis sur la situation et dans le jeu de rôles, sur le rôle à jouer. Cependant, il faut bien reconnaître qu'il n'y a pas de jeux de rôles sans situation, et pas de situation sans rôle à incarner. Je regrouperai donc ces deux activités.

L'étudiant doit, selon la situation, s'inventer un comportement et trouver les propos qui correspondent à son rôle. L'approche est donc à la fois linguistique et extralinguistique, verbale et physique. Les rôles peuvent être distribués ou choisis par les étudiants (il est important qu'ils aient envie de jouer le rôle qu'on leur propose ; notre intention n'est pas d'animer ces psychodrames). La durée de la simulation peut être libre ou imposée.

La préparation peut prendre plus ou moins de temps. Cette préparation est la clé de la réussite. Peut-être nuit-elle à la spontanéité, mais sans elle, il ne pourrait y avoir d'échanges linguistiques de qualité.

Le jeu de rôles est une méthode d'apprentissage par l'action. Le savoir devient un savoir-faire et son intégration à la personnalité donne le savoir-être. (Mucchielli 1983: 101)

La simulation peut être enregistrée ou filmée. Filmer la simulation peut gêner ou motiver les étudiants. Tout dépend de la façon dont l'enseignant présente cet aspect du travail. J'ai constaté que les étudiants manifestaient plus d'enthousiasme, les quelques premiers moments d'inquiétude passés, s'ils sont prévenus que la simulation sera filmée. Ils sont enchantés de se voir sur le petit écran. Ils me demandent de leur dupliquer la bande vidéo.

Filmer une simulation présente l'avantage inestimable de garder des traces de la simulation. On peut la visualiser plusieurs fois et s'arrêter sur l'image. Cela facilite la discussion sur les difficultés linguistiques ou extralinguistiques rencontrées. Le souvenir approximatif d'un comportement ne peut avoir la force probante d'une image.

Pendant le tournage j'interdis l'accès du studio à ceux qui ne participent pas à l'activité filmée. Comme les étudiants sont curieux de voir comment leurs camarades se sont comportés, j'organise au cours suivant une séance de visionnement pour que tous les étudiants apprécient le travail des autres et en profitent. Cela crée un événement vécu en commun. La projection du film doit être conduite de manière à ne pas mettre en situation déstructurante les étudiants qui ont accepté d'être les protagonistes d'une simulation. Voici la procédure que j'ai établie pour la relecture d'une bande vidéo : retransmission de la séquence sans commentaire ni arrêt sur image. Commentaires des protagonistes sur la simulation qu'ils ont vécue et observent. Interventions des spectateurs en anglais et uniquement sur les aspects positifs qu'ils ont remarqués. Intervention de l'enseignant au 
cours d'une nouvelle projection de la bande vidéo avec arrêt et retour sur image pour mettre en valeur un point fort ou une situation critiquable.

À la fin de cette deuxième projection, les étudiants discutent et confrontent leur point de vue en s'exprimant en anglais. Remise de la bande vidéo à l'un des acteurs de la simulation désigné par les partenaires, pour permettre à l'équipe de se revoir et d'analyser la situation en « comité restreint ».

Les thèmes de simulation sont très nombreux. Voici ceux qui ont le mieux marché dans nos cours : chez un distributeur de produits informatiques : présentation d'un produit ou vente d'un produit (logiciel ou matériel); dans une exposition: démonstration d'un produit (logiciel ou matériel); dans une entreprise : conseils sur l'informatisation des bureaux, choix d'un réseau local, installation d'un produit (logiciel ou matériel); dans un bureau de recrutement ou dans une entreprise : entretien d'embauche.

Dans tous les cas la préparation de la simulation se fait chez soi et/ou en cour à partir de documents anglais, articles, publicités, offres d'emploi, dépliants décrivant les activités des entreprises. L'enseignant insiste pour que le déroulement de la simulation soit bien structuré et le discours des étudiants, simple et clair.

Selon leur niveau, les étudiants pourront avoir recours à des notes plus ou moins rédigées.

\section{Expression libre, créativité}

174 L'expression libre est une activité qui ne peut être mise en place que lorsque les étudiants se connaissent très bien et que leur niveau en anglais est à la fois très bon et homogène. Une telle activité développe l'invention et la spontanéité dans l'expression, mais peut très vite devenir ennuyeuse et banale. Les étudiants ne savent plus quoi dire et la discussion tourne en rond.

S'il y a des lecteurs anglophones dans l'établissement, il est préférable de leur demander d'être présents pour qu'ils puissent relancer le débat. L'enseignant reste en arrière-plan, note discrètement les erreurs et les communique au groupe ultérieurement. Les groupes doivent être de petite taille pour que tout le monde puisse et ose s'exprimer.

Proposons des débats sur des sujets qu'ils connaissent bien en français: les virus informatiques, l'informatique chez soi, informatique et liberté, la musique synthétique.

Ces débats peuvent être de simples discussions à bâtons rompus ou des débats plus structurés avec un animateur interrogeant tour à tour les membres de deux équipes ayant des points de vue diamétralement opposés.

Correspondance en temps différé via l'ordinateur et téléconférence

La correspondance en temps différé via l'ordinateur et la téléconférence télématique permettent à des étudiants d'un même pays ou de différents pays de communiquer entre eux. Les messages sont transmis par ordinateur.

F. Debyser a présenté ces deux types de correspondance aux États Généraux des Langues : la première utilise le couplage ordinateur-carte MODEM-téléphone; la deuxième permet des liaisons en direct. Dans les deux cas, les coûts dépendent de la durée de la communication téléphonique.

181 F. Debyser indique que «la téléconférence peut être utilisée pour des animateurs et des créations à distance d'un type nouveau " (Debyser 1989). Il y a une véritable interaction entre des étudiants qui se trouvent à des dizaines, des centaines ou des milliers de kilomètres les uns des autres. F. Debyser insiste sur la nécessité d'avoir un scénario bien 
préparé pour que la communication ne soit pas ratée. Le rôle de l'animateur pendant les échanges est primordial. Il résume et réanime le débat.

Toutes les activités présentées dans ce chapitre peuvent être indépendantes les unes des autres. Elles peuvent aussi être cumulées et donner lieu à un projet de plus grande envergure.

\section{Projet de synthèse avec soutenance orale}

Toute réalisation concrète, qu'elle soit individuelle ou commune, stimule l'apprentissage d'une langue. Elle permet aussi de tester si les techniques de compréhension et d'expression ont été bien assimilées.

À différents moments de progression dans nos cours, il y a différentes façons de cumuler les travaux entre eux: cumul d'activités variées pour rendre plus original et plus motivant le déroulement d'une séquence d'apprentissage ; cumul pour réaliser un projet de synthèse. Sur ce sujet, nous vous conseillons aussi l'article de Locke Carter (1992).

Voici la présentation de deux projets de synthèse que nous avons expérimentés è l'IUT de Paris. Tous les deux se déroulent sur cinq semaines.

Premier projet de synthèse : travail individuel qui conduit à un rapport écrit rédigé chez soi. Les étudiants choisissent un sujet en rapport avec l'informatique. Ils recherchent un maximum d'informations en langue anglaise sur ce sujet: les documents peuvent être écrits, sonores ou visuels. Ils nous soumettent un rapport écrit de huit pages (double interligne) en anglais sur le thème choisi.

ent peut être la synthèse (c'est-à-dire reformulation) des documents en langue étrangère qu'ils possèdent sur le sujet. Ils rédigent un sommaire, un index et deux résumés : l'un en français, l'autre en anglais, d'une demi-page chacun. Ils relèvent les mots clés de leur rapport. Ils exposent leur projet au reste de la classe. Quinze minutes par projet: dix minutes pour la présentation et cinq minutes pour répondre aux questions du groupe-classe. Les thèmes étant différents, le groupe-classe prend des notes et profite ainsi des recherches faites par chaque étudiant.

Deuxième projet de synthèse $e^{4}$ : travail collectif et individuel qui se termine par un essai rédigé dans des conditions d'examen. C'est un travail de recherche qui se prépare en cours et hors cours. Travaux écrits et oraux se succèdent en alternance.

Les étudiants (par groupe de cinq environ) recherchent des articles sur un même thème lié à l'informatique. Ils exploitent ces articles (deux à trois pages par étudiant) en réalisant des fiches de vocabulaire écrites et en préparant des résumés qui seront présentés oralement au reste du groupe-classe réuni. Chaque étudiant a ensuite l'obligation de lire attentivement tous les articles qui ont trait à un même thème et de retenir le vocabulaire et les idées présentés. L'enseignant s'efforce de trouver un sujet qui exige la synthèse de l'ensemble des articles sur un même thème et le propose aux étudiants pour qu'ils rédigent en temps limité et sans document, un essai, en anglais, d'environ six cents mots.

Ces deux projets sont motivants : ils responsabilisent les étudiants - ce sont eux qui décident du thème sur lequel ils vont travailler et qui sélectionnent les articles qu'ils auront à exploiter et à assimiler ; ils renforcent et complètent les connaissances acquises ; ils transforment les relations apprenant/apprenant et enseignant/apprenant - les étudiants s'entraident et l'enseignant joue le rôle de consultant, se rendant disponible 
tour à tour pour tous ceux qui le sollicitent. Je préfère le deuxième projet pour les raisons suivantes.

Il est plus équitable. Pendant la préparation du projet, j'incite fortement les étudiants à se trouver des alliés, comme ils devront le faire en entreprise. Ensuite je leur montre que dans la vie, on est seul, face à une copie, à une décision et que, de ce fait, en phase finale, il est nécessaire de rendre un travail individuel.

Il permet une meilleure mémorisation. Pour être capable de rédiger, seul, un essai sur un sujet spécifique, il faut l'avoir consciencieusement préparé.

Trop d'étudiants se faisaient aider par des amis bien intentionnés lorsque le rapport pouvait se terminer chez soi. Certains ne prenaient même pas le temps de s'imprégner de ce qu'amis ou parents avaient rédigé. Ils rendaient un rapport impeccable (langue et présentation), mais ils n'avaient en aucun cas amélioré leurs connaissances linguistiques sur ce sujet.

La règle du jeu du deuxième projet de synthèse est bien acceptée par les étudiants, car elle respecte leur capacité à se préparer à une situation et valorise leur aptitude à y faire face seuls.

La plupart des activités peuvent être réalisées en groupe. Les projets de fin de formation sont individuels, mais le travail de préparation se fait en équipe. Dans certains cas, comme la simulation, l'expression libre ou la télécommunication, il est indispensable de travailler en équipe. Je suis particulièrement favorable au travail collectif, car il présente des avantages indéniables.

\section{Travail en groupe}

Nos cours sont composés au maximum de quinze étudiants. Il nous est donc possible, selon les travaux à accomplir, de faire éclater ce premier groupe en divers sous-groupes. Le groupe minimal étant la paire.

197 Chaque groupe peut avoir une tâche identique ou des tâches différentes à accomplir. La composition des groupes n'est jamais évidente. Je pense qu'il est préférable que les étudiants déterminent les groupes en fonction de leur personnalité et de leurs amitiés ainsi qu'en fonction des tâches proposées. En début d'année lorsqu'ils ne se connaissent pas, on leur propose de s'allier avec la personne qui se trouve placée à côté d'eux.

En université ou dans des cours pour adultes, les problèmes interrelationnels entre deux individus sont exceptionnels. Si ce problème se posait, le rôle de l'enseignant serait d'intégrer la personne rejetée ou ignorée par les autres. Les groupes se forment, souvent les garçons d'un côté et les filles de l'autre. C'est dommage! Nous les incitons à combiner leurs compétences et leurs styles.

Les tâches doivent être conçues pour stimuler l'ensemble du groupe. Un travail jugé inutile ou ennuyeux démotivera le groupe et lassera l'étudiant le plus impliqué. Il est important de prévoir des corrigés pour tous les types d'activités. Tout travail non corrigé perd de son intérêt. La réussite d'un groupe accroît sa cohésion et l'incite à obtenir des résultats encore plus performants.

L'efficacité d'un groupe semble liée aux trois composantes suivantes: l'implication, la valorisation et la compétition. Pour qu'il fonctionne bien, il est nécessaire que ces trois composantes se combinent, sans qu'il y ait domination de l'une par rapport aux autres. 
L'implication permet de s'intéresser à ce que l'on fait et d'être particulièrement motivé. La valorisation permet de prendre confiance en ses propres capacités. La compétition donne envie d'aller plus loin, de se dépasser.

Parfois nous rencontrons des étudiants réticents, ou même récalcitrants qui préfèrent travailler individuellement, tant ils sont habitués à être seuls. Ils se rendront très vite compte que la co-réflexion et les co-décisions sont fructueuses, que lorsque les forces s'ajoutent elles sont plus puissantes et qu'aider les autres apporte une véritable satisfaction et un sentiment de responsabilité accru.

L'enseignant veillera à ce que dans les différentes phases de travail (préparation, compréhension, réflexion, entraînement, transfert) chaque groupe et à chaque membre du groupe soient concernés.

Même le groupe le plus faible peut être valorisé si l'enseignant lui en donne les moyens et s'il a su rester à son écoute.

\section{Conclusion}

Les matériaux et les activités linguistiques que je viens d'énumérer permettent à tout enseignant de concevoir des cours efficaces et de qualité. Mais l'enseignant a encore un rôle primordial à jouer. Sans charisme, sa compétence ne sera qu'en partie appréciée et le message qu'il veut faire passer ne sera pas totalement transmis.

Qu'est-ce que le charisme ? C'est un mélange composite qui combine à doses difficiles à normaliser : compétence, équité, préoccupation et respect de l'autre, joie d'enseigner, joie de découvrir, enthousiasme, gaîté, bonne humeur, honneur de donner, bonheur de recevoir, dynamisme, ouverture d'esprit, sens de l'humour non déstabilisateur et esprit de répartie non castrateur, pas de prétention, pas de pédanterie, chaleur, confiance, une certaine fantaisie (pour souligner la compétence et non pour masquer l'incompétence), un certain non-conformisme pour développer chez l'étudiant la liberté créatrice.

Rappelez-vous du film Le cercle des poètes disparus ${ }^{5}$. Il faut qu'il y ait du John Keating dans chacun de nous. Lorsque l'on m'interroge sur mon métier et que je réponds que j'enseigne l'anglais de l'informatique, mes interlocuteurs (collègues enseignant l'anglais général ou amis ayant un tout autre métier), me témoignent de la commisération. Comme cela doit être triste!

Cette communication devrait les convaincre du contraire.

Enseigner une langue de spécialité est enrichissant et est passionnant; c'est un monde toujours inachevé, celui de nos futurs, et cela peut être gai.

Nous avons à notre disposition: des matériaux excellents qui intéressent nos étudiants parce qu'ils sont liés à leur future profession; des matériaux qui nous rendent compte des dernières découvertes technologiques ainsi qu'un choix pratiquement illimité d'activités didactiques qui permettent de varier les approches et les animations de chaque cours.

210 J'ai la certitude que nous apprenons à nos étudiants quelque chose d'utile. Notre relation avec eux est enrichissante : ils nous aident parfois à comprendre certains domaines que nous n'avons pas encore eu le temps d'explorer à fond. Notre relation avec les enseignants de leur discipline principale, l'informatique est, elle aussi privilégiée : nous travaillons pour eux, ils sont disposés à nous aider - les cours d'anglais et d'informatique 
se complètent. Comment ne pas éprouver du plaisir, comment ne pas communiquer et partager ce plaisir?

211 Le concept de langue de spécialité est difficile à définir. Est langue de spécialité la langue d'un métier, mais aussi la langue liée à un domaine particulier : langue de la politique, d'un sport, d'un jeu... Quand nous préparions le CAPES et l'agrégation, n'étions-nous pas déjà en train d'apprendre des langues de spécialité en découvrant le vocabulaire de la mer, des navires, des forêts et des champs... ?

La stratégie présentée dans cette communication peut s'appliquer à toute autre langue de spécialité. Elle permet de choisir matériaux linguistiques et activités didactiques qui facilitent et dynamisent l'apprentissage d'une langue. L'apprentissage sera d'autant plus efficace que les matériaux seront clairs, simples, concrets et structurés et les activités et les outils didactiques utilisés, variés.

Matériaux linguistiques et activités didactiques apportent pertinence et cohérence à nos cours. Par son charisme l'enseignant y apporte enthousiasme et gaîté. Les motivations de chacun sont intensifiées, la réussite facilitée.

\section{BIBLIOGRAPHIE}

Carter, Locke. 1992. « Computer-mediated discourse and student participation ». Cahiers de l'APLIUT 45, 66-76.

Debyser F. 1989. « La télématique : implications et conséquences pour l'enseignement et l'apprentissage des langues ». Paris : Les États Généraux des Langues.

Dechet, Arlette. 1990. «Stratégie pour l'enseignement de l'anglais de l'informatique : démarche didactique intégrant l'ordinateur et le traitement de texte ». Thèse de doctorat soutenue à Université Paul Valéry Montpellier 3, sous la direction de Jean-Marie Baïssus.

Dechet, Arlette. 1991. «Le traitement de texte WORD (version anglaise) : interface privilégiée pour la conception de cours d'anglais de spécialité ». Bulletin trimestriel EPI (association Enseignement Public et Informatique) 61, 133-148.

Dechet, Arlette. 1991. " Projet de fin de formation en anglais de l'informatique ». Les Cahiers de l'APLIUT 42-43, 41-55.

Dubois, Jean. 1973. Dictionnaire de linguistique. Paris : Larousse. Perrin, Michel. 1990. « De l'utilisation 'communicative' des documents authentiques ». Actes $d u X^{e}$ colloque du GERAS, Bordeaux, 9-33.

Mucchielli, Alex. 1983. Les jeux de rôles. Paris : Presses universitaires de France, Que sais-je ? Pradeilles, Anne. 1981. «A new C.A.R. for ESP teachers : quelques modestes propositions pour la recherche assistée par ordinateur ». Les Cahiers de l'APLIUT 2, 91-100.

Vinay, J.-P. et J. Darbelnet. 1977. Stylistique conparée du français et de l'anglais, Méthode de traduction. Paris : Didier.

Yaguello, Marina. 1981. Alice au pays du langage. Paris : Seuil. 


\section{Manuels d'informatique}

Athey Thomas H. \& Robert W. Zmud. 1988. Introduction to Computers and Information Systems with Basic. Second ed. Glenview, IL : Scott, Foresman and Company, 540 pages.

Bishop, Peter. 1984. Computing Science. Walton-on-Thames : Nelson, 1984, 362 pages.

Brookes, Michael et François, Lagoutte. 1989. English for Information Technology. Paris : Belin, 223 pages.

Dechet, Arlette. 1988. GOTO, L'anglais informatique pour communiquer. Paris : Ellipses, 240 pages.

Gallet, Liliane. 1981. L'anglais pour informaticien. Paris : Cedic/Fernand Nathan, 324 pages.

Gallet, Liliane et Jean Brossard. 1990. L'anglais pour l'informatique. Paris : Dunod Informatique, Bordas, 308 pages.

Glendinning Eric H. \& John McEwan. 1987. English in Computing. Londres : Unwin Hyman Limited, 128 pages.

Goldschlager Les \& Andrew Lister. 1988. Computer Science “A Modern Introduction”. Second ed. New York : Prentice Hall, 330 pages.

Humby Edward \& Philip Robinson. 1980. Computer Applications. Special English. Londres : Cassell, 124 pages.

Humby Edward \& Philip Robinson. 1981. Computers. Special English. Londres : Cassell, 119 pages.

Leray, Y., A. Pradeilles et N. Vigouroux-Frey. 1980. This Quiet Revolution. Paris : Armand Colin-

Longman, 178 pages.

Mandell, Steven L. 1985. Computers and Data Processing "Concepts and applications". Third Edition. Saint Paul : West Publishing Company, 512 pages.

Mullen D. Norma \& P. Charles Brown. 1987. English for Computer Science. New Edition revised and updated. Oxford : Oxford University Press, 241 pages.

Meyers, Richard A. 1984. Computer Science. New York : Macmillan Publishing Company.

Robinson, Philip.1981. Computer Programming. Special English. Londres : Cassell, 104 pages.

\section{NOTES}

1. Dans Le Robert, bidouiller signifie «faire fonctionner, arranger en bricolant, faire fonctionner ingéniosité, de façon improvisée». Pour les enseignants d'informatique, ce terme a un sens négatif. Ils qualifient de bidouilleurs les étudiants qui ont touché aux ordinateurs, qui croient et prétendent savoir s'en servir mais qui n'ont aucune démarche structurée pour analyser et résoudre un problème informatique. Le bidouilleur sera donc méprisé par certains pour son manque de professionnalisme et adulé par d'autres pour son ingéniosité.

2. Thérèse Brouat prépare actuellement un DEA sur les publicités informatiques sous la direction de Monsieur le Professeur Hubert Greven. La communication qu'elle fera au XIII ${ }^{\mathrm{e}}$ colloque du GERAS et qui devrait être publiée dans les Actes du treizième colloque du GERAS 1992, nous aidera à mieux utiliser les publicités informatiques pendant nos cours.

3. «Finding a virus can be a hare raising experience ». Dr. Solomon's Anti-Virus Toolkit. Ontrack Computer Systems, 6321 Bury Drive, Eden Prairie, MN 55346. 
4. Voir à ce sujet Dechet, A. 1991. "Projet de fin de formation en anglais de l'informatique ». Communication aux Journées des enseignants d'anglais des départements informatiques des IUT, Reims février 1991, publiée dans Dechet (1991).

5. Le cercle des poètes disparus (The Dead Poets' Society) est un film américain de Peter Weir (1989) avec Robin Williams dans le rôle de John Keating, le professeur de littérature anti-conformiste qui enseigne avant tout la liberté de penser et la passion de la poésie.

\section{AUTEUR}

\section{ARLETTE DECHET}

IUT Paris 5 